\title{
Keloids and hypertrophic scars in secondary school in the city of Ouagadougou (Burkina Faso)
}

\section{Adama Traoré1,2, Nina Korsaga/Somé1,3, Amina Zoungrana/Ouédraogo ${ }^{1,2}$, Nayi Zongo1, Gilbert Patrice Tapsoba', ${ }^{1,2}$ Muriel Ouédraogo/Ouédraogo',2, Prisca N'Dah'2, Jean-Baptiste Andonaba4 ${ }^{4}$, Fatou Barro/Traoré', Pascal Niamba ${ }^{1,2}$}

${ }^{1}$ Faculty of Health Science of, Joseph Ki-Zerbo University, Ouagadougou, Burkina Faso, ${ }^{2}$ Department of Dermatology and Venerology, Yalgado Ouédraogo University Hospital of Ouagadougou, Burkina Faso, ${ }^{3}$ Department of Dermatology and Venereology of Boulmiougou District Hospital, Ouagadougou, Burkina Faso, ${ }^{4}$ Higher Institute of Health Sciences, Nazi Boni University, Bobo-Dioulasso, Burkina Faso

Corresponding author: Dr. Nina Korsaga/Somé, E-mail: nessine2000@yahoo.fr

\begin{abstract}
Introduction: Keloids and/or hypertrophic scars (ch/CH) are benign tumors, poorly understood and studied, yet with socio-economic impact for serious cases precisely to African black people in less developed countries. We wanted to evaluate their frequency and epidemiological and clinical aspects in secondary school in the city of Ouagadougou. Patients and methods: It was a descriptive cross-sectional study, from March 6 to April 22 2017, on secondary school students from the city of Ouagadougou. We used the stage-three random sampling technique from the school population list from the city of Ouagadougou in 2016-2017. The sample minimal size was 904 according to Pascal Ardilly Formula. The sought variables were epidemiological, clinical and therapeutic. Variables on the impact and students' knowledges about ch/CH scars were also sought. Results: Out of 1225 examined students, we identified $125 \mathrm{ch} / \mathrm{CH}$ scars carriers, that's a frequency of $10,20 \%$. There were 99 cases of keloids (79,2\%), 26 cases of hypertrophic scars (20,8\%) and no case of keloid diseases. Depending on the sex, there were 64 girls, and 61 boys. The average age was 18,10 years. $22,4 \%$ of the cases presented a family history for ch/CH scars. The traumatic etiology was found in $96 \%$ of the cases mostly with injuries made by sharp objects $(48 \%)$, and public road accidents $(28,3 \%)$. Therapeutic attitudes were abstention $(78,4 \%)$, traditional treatment $(18,4 \%)$ and modern treatment $(3,2 \%)$. An aesthetic implant was found to 24 students including 17 girls. According to the student, ch/CH scar was a healing anomaly (484 students), a malformation (95), a bewitchment (20), a type of cancer (2). Conclusion: Keloids/Hypertrophic scars frequency is high to school student in the city of Ouagadougou. The lack of medical care is probably linked to students' ignorance about possibilities of support.
\end{abstract}

Key words: Keloids and/or hypertrophic; Students; City of Ouagadougou

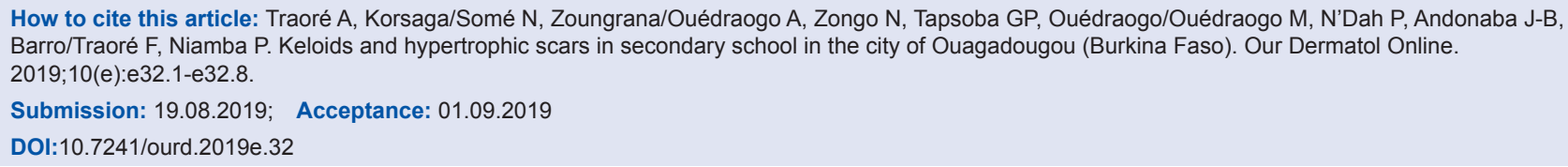




\title{
Cicatrices chéloïdes et hypertrophiques en milieu scolaire secondaire de la ville de Ouagadougou (Burkina Faso)
}

\author{
Adama Traoré1,2, Nina Korsaga/Somé1,3, Amina Zoungrana/Ouédraogo ${ }^{1,2}$, Nayi Zongo1, \\ Gilbert Patrice Tapsoba',2, Muriel Ouédraogo/Ouédraogo',2, Prisca N'Dah², \\ Jean-Baptiste Andonaba ${ }^{4}$, Fatou Barro/Traoré', Pascal Niamba ${ }^{1,2}$
}

${ }^{1}$ Faculty of Health Science of, Joseph Ki-Zerbo University, Ouagadougou, Burkina Faso, ${ }^{2}$ Department of Dermatology and Venerology, Yalgado Ouédraogo University Hospital of Ouagadougou, Burkina Faso, ${ }^{3}$ Department of Dermatology and Venereology of Boulmiongou District Hospital, Ouagadougou, Burkina Faso, ${ }^{4}$ Higher Institute of Health Sciences, Nazi Boni University, Bobo-Dioulasso, Burkina Faso

Corresponding author: Dr. Nina Korsaga/Somé, E-mail: nessine2000@yahoo.fr

\begin{abstract}
RÉSUMÉ
Introduction: Les chéloïdes et ou cicatrices hypertrophiques $(\mathrm{ch} / \mathrm{CH})$ sont des tumeurs bénignes mal connues, peu étudiées et ayant pourtant un impact socio-économique dans les formes graves notamment chez les noirs africains des pays peu développés. Nous avons voulu évaluer leur fréquence et leurs aspects épidémiologiques et cliniques en milieu scolaire secondaire de la ville de Ouagadougou. Patients et méthodes: Il s'agissait d'une étude transversale descriptive du 6 mars au 22 avril 2017, portant sur les élèves des établissements d'enseignement secondaire de la ville de Ouagadougou. Nous avons utilisé la technique d'échantillonnage aléatoire à trois degrés à partir de la liste et des effectifs des 508 établissements d'enseignement secondaire général et/ou technique de la ville de Ouagadougou en 2016-2017. La taille minimale de l'échantillon était de 904 selon la formule de Pascal Ardilly. Les variables recherchées étaient épidémiologiques, cliniques et thérapeutiques. Des variables sur le retentissement et les connaissances des élèves sur les cicatrices ch/CH étaient également recherchées. Résultats: Sur un total de 1225 élèves examinés, nous avons recensé 125 porteurs de cicatrices ch/CH, soit une fréquence de 10,20\%. Il y avait 99 cas de chéloïdes (79,2\%), 26 cas de cicatrices hypertrophiques $(20,8 \%)$ et aucun cas de maladie chéloïdienne. Selon le sexe on dénombrait, 64 filles. Lâge moyen des élèves était de 18,10 ans. Un antécédent familial de cicatrices $\mathrm{ch} / \mathrm{CH}$ était retrouvé dans 22,4\% des cas. L'étiologie traumatique était retrouvée dans $96 \%$ des cas avec surtout les blessures par objet tranchant (48\%) et par accidents de la voie publique (AVP) (28,3\%). Les attitudes thérapeutiques étaient l'abstention $(78,4 \%)$, le traitement traditionnel $(18,4 \%)$ et le traitement moderne $(3,2 \%)$. Un retentissement esthétique était retrouvé chez 24 élèves dont 17 filles. Pour les élèves la cicatrice chéloïde/hypertrophique était une anomalie de la cicatrisation (484 élèves), une malformation (95), un envoutement (66), une malédiction (20) ou un type de cancer (2). Conclusion: La fréquence des cicatrices chéloïde/hypertrophiques est élevée chez les élèves en milieu scolaire de la ville de Ouagadougou. Labsence de recours aux soins médicaux est probablement liée à l'ignorance des élèves sur les possibilités de prise en charge.
\end{abstract}

Mots clefs: Cicatrices chéloïdes/hypertrophiques; Élèves; Ouagadougou

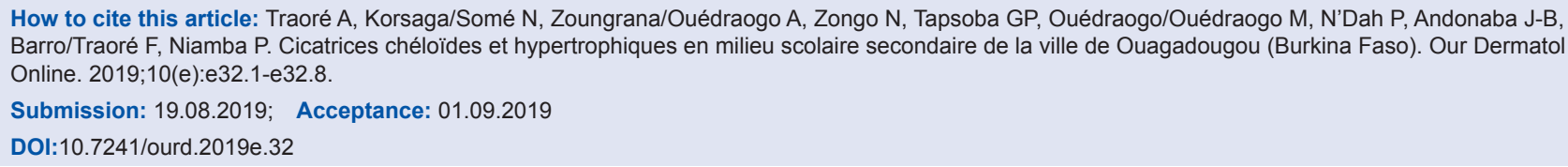




\section{INTRODUCTION}

Les cicatrices chéloïdes et hypertrophiques sont des tumeurs bénignes intradermiques spontanées ou acquises, correspondant à une réaction inappropriée du tissu conjonctif à un traumatisme parfois minime, entrainant accumulation de collagène de type I dans le derme, chez des personnes prédisposées [1-5]. Dans les formes acquises elles peuvent apparaitre après divers traumatismes (brûlure, piercing, tatouage, rasage, morsure, scarification, vaccination, lésions de grattage), après un acte chirurgical, ou être secondaires à une affection dermatologique (acné, varicelle, zona, folliculite) $[2,6]$. Elles sont plus fréquentes chez les personnes à peau noire originaires de l'Afrique et du Sud de l'Inde et plus rares chez le caucasien et l'albinos [4,5,7-10]. Les premières descriptions de cette affection sont attribuées à Noël Retz en 1790 et à Louis Alibert en 1806 qui parlaient respectivement de «hernies graisseuses et de« cancroïde" $[11,12]$. Quant à la cicatrice hypertrophique, elle présente le même aspect de départ mais ne s'étend pas en périphérie. Elle est toujours secondaire à un traumatisme, apparait après une durée d'environ un mois et évolue spontanément vers la régression en 12 à 18 mois [2].

Il existerait chaque année, 11 millions de chéloïdes dans le monde [13]. En Europe, on note une prévalence de cas de cicatrices chéloïdiennes comprise entre 6 et $16 \%$, avec une nette prédominance du sujet à peau noire $[1,14]$. En Afrique Noire ou ces pathologies prédominent les données restent insuffisantes, parcellaires et surtout hospitalières. Les fréquences hospitalières suivantes sont notées: 2,5\% au Congo [15], 1,9\% au Togo [16], 1,2\% au Sénégal [17] et 0,7\% au Bénin [18].

Au Burkina Faso une fréquence hospitalière de 3,5\% était notée en 2014 dans le service de dermatologie/ vénéréologie du centre hospitalier universitaire Yalgado OUEDRAOGO (CHU-YO) [19] et ces cicatrices constituaient un des motifs de plus en plus fréquents de consultation. Labsence de données épidémiologiques en population générale, dans nos régions, sur ces affections, rend leur prévention et leur prise en charge plus difficile pour le praticien.

Le but de ce travail était d'étudier en milieu scolaire les aspects épidémiologiques, cliniques, thérapeutiques et sociologiques des ch/CH dans une population jeune.

\section{MATERIALS AND METHODS}

\section{Cadre De L'étude}

Leétude s'était déroulée à Ouagadougou, capitale politico-administrative du Burkina Faso. Cette capitale comptait 2753852 habitants avec un taux d'accroissement de 9,3\% par an [20], 508 établissements d'enseignement secondaire général et/ou technique et 211167 élèves (47\% de garçons et 53\% de filles) [21] durant la période de l'étude.

\section{Type, Période D’étude Et Échantillonnage}

Nous avons réalisé une enquête transversale de type descriptif en un seul passage, sur une période de 7 semaines, allant du 06 mars au 22 avril 2017. Léchantillonnage à trois degrés était le type choisi. La prévalence de l'affection en population scolaire étant méconnue, la taille était calculée selon un taux de prévalence de $50 \%$, et une marge d'erreur de $5 \%$, selon la formule de Pascal Ardilly:

$$
n \geq(Z x)^{2} \times \frac{[p(1-p)]}{m^{2}} \times \frac{1}{t} \times e f f
$$

$[n=$ taille minimale de l'échantillon; $x=$ niveau de confiance (fractile de la loi normale avec $Z x=1,96$ ); $p=$ prévalence de l'affection dans la population, $50 \% ; m=$ marge d'erreur $(m=0.05)$; eff $=$ effet de sondage $(\mathrm{eff}=2) ; t=$ taux de réponse $(\mathrm{t}=85 \%)]$.

Nous avons inclus, tout élève régulièrement inscrit dans un des établissements secondaires (indépendamment du sexe, de l'âge et du type d'établissement), présent durant la période d'étude, tiré au sort et ayant donné son consentement éclairé.

\section{Population Étudiée}

Nous avons sélectionné 9 établissements, 49 classes et 1225 élèves dont 700 du premier cycle et 525 du second cycle.

\section{RESULTS}

\section{Pour Ce Qui Était Des Aspects Épidémiologiques}

La fréquence: nous avons colligé 125 cas de chéloïdes et/ou cicatrices hypertrophiques (ch/CH) sur 1225 élèves examinés, soit une fréquence globale de 10,2\%. 
La fréquence des chéloïdes était de 8,1\% (99 cas) et celle des cicatrices hypertrophiques de 2,1\% (26 cas).

Lâge: l'âge moyen des élèves porteurs de l'affection était de 18,1 ans avec une prédominance de la tranche d'âge des élèves de 15 à 20 ans (Tab. 1).

Le sexe: les élèves de sexe masculin étaient au nombre 569 et ceux de sexe féminin, au nombre de 656, soit un sex-ratio de 0,87 . Il n'y avait pas de différence statistiquement significative pour les taux de prévalence calculés en fonction du sexe et du cycle d'étude. Les ch/CH prédominaient dans la tranche d'âge des 20-25 ans.

\section{Concernant Les Données Cliniques}

Les aspects morphologiques: sur le plan du diagnostic morphologique, nous avons recensé 99 (79,2\%) cas de chéloïdes et 26 (20,8\%cas) de cicatrices hypertrophiques (Fig. 1). Nous n'avons eu aucun cas de maladie chéloïdienne.

Les facteurs déclenchant: un traumatisme était retrouvé dans 120 cas (Tab. 2) et aucune étiologie n'était retrouvée dans 5 cas. Les types de traumatisme les plus r-etrouvés étaient la perte de substance par coups et blessures volontaires (48\%), suivie des accidents de la voie publique $(27,2 \%)$. Les piercings de l'oreille étaient incriminés dans 3,2\% des cas.

Les signes fonctionnels: on notait le prurit (46 élèves) et la douleur (8 élèves).

La durée d'évolution moyenne des lésions était de 3,69 ans avec une durée minimale d'un mois et une durée maximale de 17 ans.

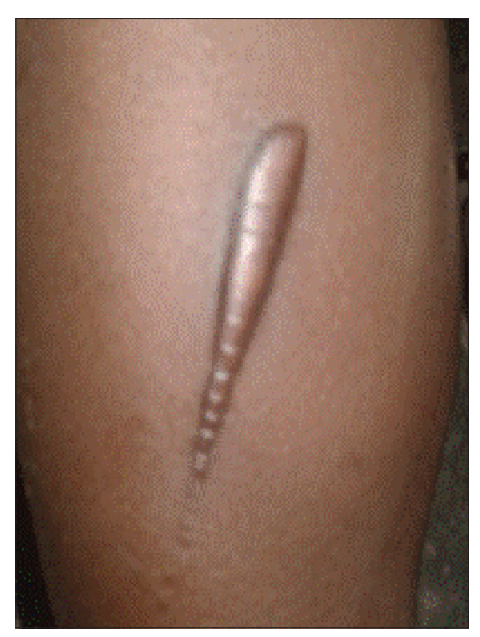

Figura 1: Cicatrice hypertrophique du mollet gauche post blessure par pédale de vélo.
L'antécédent familial de chéloïde/cicatrice hypertrophique était retrouvé chez 28 élèves.

Les données sémiologiques physiques.

Pour la couleur, les lésions étaient normochromiques dans $49,6 \%$ des cas, hyperchromiques dans $44,8 \%$ des cas et hypochromiques dans $5,6 \%$ des cas.

La surface était lisse $(87,2 \%)$, bosselée $(9,6 \%)$, rugueuse $(2,4 \%)$ ou ulcérée $(0,8 \%)$.

Les formes prédominantes étaient ovalaires $(45,6 \%)$, linéaires $(28,8 \%)$ et arrondies $(22,4 \%)$.

Les limites étaient nettes dans $92 \%$ des cas et floues dans $8 \%$ des cas.

La bordure était régulière dans $84 \%$ des cas (105 lésions) et irrégulière dans $16 \%$ des cas (20 lésions).

La taille moyenne des lésons était de $3,55 \mathrm{~cm}$ avec des extrêmes de 1 et $30 \mathrm{~cm}$. Les tailles comprises entre 2 et $4 \mathrm{~cm}$ représentaient $52 \%$ des lésions.

La consistance était ferme dans $96 \%$ des cas et molle dans $4 \%$ des cas.

Pour le nombre des lésions, les ch/CH étaient uniques dans $84,8 \%$ des cas et multiples dans $15,2 \%$ des cas (avec 2 à 3 lésions).

La localisation sur les membres supérieurs était retrouvée dans $42,4 \%$ des cas, suivie des membres inférieurs $(38,4 \%)$ et du tronc $(9,6 \%)$ (Tab. 3). Aucune lésion n'était retrouvée au niveau cuir des paumes, des plantes et des muqueuses. Les parties génitales n’étaient pas examinées.

\section{Aspects Thérapeutiques}

Nous avons noté que 27 élèves avaient entrepris un traitement, dont un traitement traditionnel dans 23 cas et un traitement moderne dans 4 cas. Le traitement moderne consistait en des infiltrations de corticoïdes ou en l'application de dermocorticoïdes prescris par un personnel médical ou paramédical sur une durée moyenne de trois mois. Quant au traitement traditionnel, il consistait surtout en l'application de la sève de bois chaud sur la lésion. Tous les élèves ayant entrepris un traitement moderne obtenaient un affaissement partiel ou total de la lésion, et tous ceux ayant entrepris un traitement 
traditionnel signalaient un aspect inchangé de la lésion, de même qu'une absence de surinfection ou d'autre complication.

\section{Aspects Sur Le Retentissement Et Les Connaissances Des Élèves Sur $\mathrm{La} \mathrm{Ch} / \mathrm{CH}$}

Sur le plan fonctionnel, les lésions étaient non gênantes pour $78,4 \%$ des élèves peu gênantes $(18,4 \%)$ et très gênantes pour 3,2\% d'entre eux. Un retentissement esthétique était retrouvé chez 24 élèves dont 17 filles. Les élèves qui avaient déjà vu ou entendu parler des ch/CH autour d'eux par les parents, les amis ou même par un agent de santé étaient au nombre de 712 (58,1\%). Pour 484 (39,5\%), la ch/CH était une anomalie de la cicatrisation, alors que 558 élèves $(45,55 \%)$ n'avaient aucune idée de ce qu'était une ch/CH. Pour d'autres, il s'agissait d'un envoutement, d'une malformation ou encore d'une malédiction (Tab. 4). Pour 767 élèves, les ch/ $\mathrm{CH}$ pouvaient se soigner. Les connaissances sur les modalités de traitement chez ces 767 élèves étaient variées, mais venaient en tête l'exérèse chirurgicale, l'ignorance et le traitement traditionnel (Tab. 5).

Tableau 1: Répartition des 1225 élèves selon la tranche d'âge

\begin{tabular}{lcc}
\hline Age group (year) & Number of student & Proportion (\%) \\
\hline$[10-15]$ & 248 & 20.2 \\
{$[15-20]$} & 735 & 60.0 \\
{$[20-25]$} & 237 & 19.4 \\
{$[25-30]$} & 005 & 0.4 \\
$>30$ & 00 & 0.0 \\
Total & 1225 & 100.0 \\
\hline
\end{tabular}

Tableau 2: Répartition des 120 cas de ch/CH selon le type de traumatisme

\begin{tabular}{lcc}
\hline Type of traumatism & $\begin{array}{c}\text { Number of } \\
\text { students }\end{array}$ & $\begin{array}{c}\text { Percentages } \\
\text { (\%) }\end{array}$ \\
\hline Loss of substance due to intentional & 60 & 50.0 \\
assault and battery [Fig. 2] & & \\
Public road accident [Fig. 3] & 34 & 28.3 \\
Thermal burns [Fig. 4] & 14 & 11.7 \\
Piercing [Fig. 5] & 4 & 3.3 \\
Chickenpox & 3 & 2.5 \\
Vaccination & 3 & 2.5 \\
Surgical incision & 2 & 1.7 \\
Total & 120 & 100.0 \\
\hline
\end{tabular}

Tableau 3: Répartition des 125 cas de ch/ $\mathrm{CH}$ selon le siège

\begin{tabular}{lcc}
\hline Injury site & Number of students & Percentages (\%) \\
\hline Upper limbs & 53 & 42.4 \\
Lower limbs & 48 & 38.4 \\
Torso & 12 & 9.6 \\
Cephalic ends & 11 & 8.8 \\
Pubis & 1 & 0.8 \\
Total & 125 & 100.0 \\
\hline
\end{tabular}

\section{DISCUSSION}

Il s'agit à notre connaissance d'une des premières études de prévalence sur la maladie en milieu scolaire en Afrique noire. Cette étude, malgré les limites inhérentes à certaines données recueillies à l'interrogatoire, nous a donné des chiffres fiables sur les ch/CH dans le milieu scolaire, du fait sa méthodologie et de la population étudiée.

\section{Données Épidémiologiques}

Les résultats de ce travail ont montré une prévalence élevée des ch/CH en milieu scolaire secondaire de la ville de Ouagadougou. Ce résultat était très proche de celui admis par la littérature. En effet les différentes études notent que les chéloïdes sont présentes dans toutes les ethnies avec une prévalence générale estimés entre 4,5 et $14 \%$ et pouvant dépasser $14 \%$ dans la population africaine $[2,22]$.

Les résultats des études hospitalières en milieu Africain montrent des fréquences généralement plus faibles allant de 0,4 à $2,5 \%$ selon les pays et démontrent que cette méthode sous-évalue ces pathologies [15-18,23]. Le biais de recrutement, le faible recourt aux centres de santé pour multiples raisons expliquent cette sous-évaluation.

La fréquence plus élevée des ch/CH dans la tranche des 18-24 ans confirmait les données selon lesquelles

Tableau 4: Répartition des 1225 élèves selon leur définition des $\mathrm{ch} / \mathrm{CH}$

\begin{tabular}{lcc}
\hline Ch/CH scars definition & $\begin{array}{c}\text { Number of } \\
\text { students }\end{array}$ & Percentages (\%) \\
\hline No idea & 558 & 45.5 \\
Healing anomaly & 484 & 39.5 \\
Malformation & 95 & 7.8 \\
Bewitchment & 66 & 5.4 \\
Curse & 20 & 1.6 \\
Type of cancer & 02 & 0.2 \\
Total & 1225 & 100.0 \\
\hline
\end{tabular}

Tableau 5: Répartition des 767 élèves selon leur connaissance sur le traitement des $\mathrm{ch} / \mathrm{CH}$

\begin{tabular}{lcc}
\hline $\begin{array}{l}\text { Knowledges about the } \\
\text { treatment }\end{array}$ & $\begin{array}{c}\text { Number of } \\
\text { students }\end{array}$ & Percentages (\%) \\
\hline Exerisis & 294 & 38.4 \\
No idea & 280 & 36.5 \\
Traditional treatment & 83 & 10.8 \\
Injections & 50 & 6.5 \\
Plastic surgery & 16 & 2.1 \\
Cream & 16 & 2.1 \\
Medicine with no precision & 14 & 1.8 \\
Prayer sessions & 14 & 1.8 \\
Total & 767 & 100.0 \\
\hline
\end{tabular}




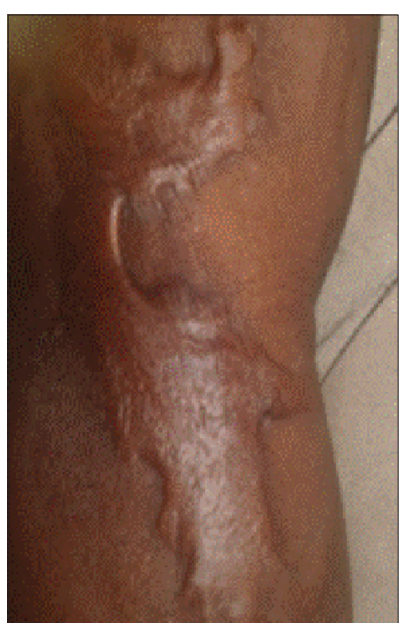

Figura 2: Chéloïde du membre supérieur gauche par coup et blessure volontaire.

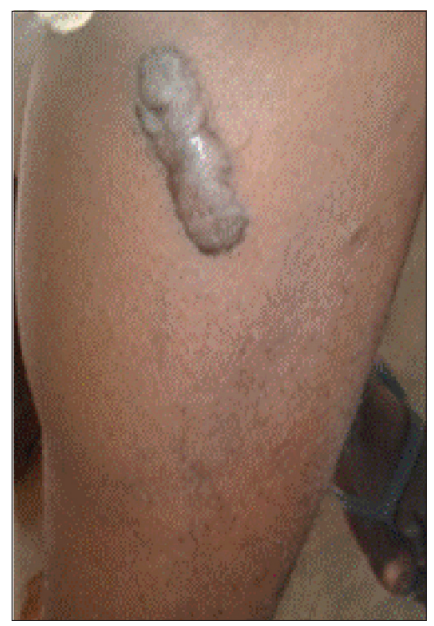

Figura 3: Chéloïde du tiers supérieur de la jambe gauche post AVP.

les ch/CH surviennent dans les 3 premières décennies de la vie, elles sont donc plus rares chez les sujets très jeunes et chez les sujets très âgés [7].

\section{Données cliniques}

La prédominance des traumatismes par coups et blessures volontaires et accidents de la voie publique comme facteurs déclenchant se comprend dans cette population jeune et active, plus sujette aux jeux dangereux et aux chutes. De plus dans notre contexte les moyens de déplacement favori des lycéens sont les engins à deux roues avec leur corollaire d'AVP liés le plus souvent au non-respect du code de la route et à la vitesse pratiquée par ces jeunes. Les ch/CH étaient peu prurigineuses dans cette population. Les personnes à peau noire originaires de l'Afrique et du Sud de l'Inde semblent les plus fréquemment atteintes [4,5,7-10]. La prédisposition familiale retrouvée dans $1 / 4$ des cas corroboraient les données de la littérature.

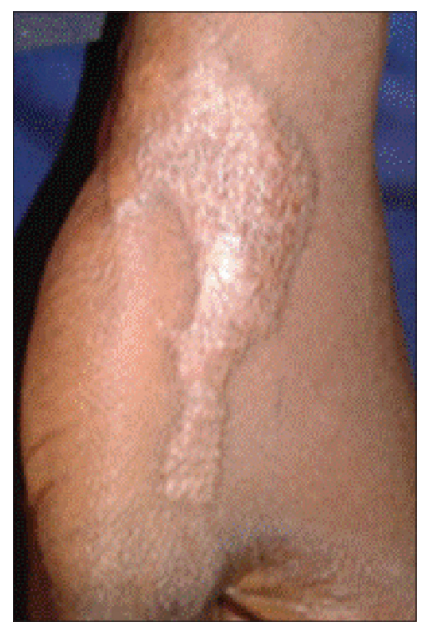

Figura 4: Chéloïde du dos de la main gauche post brûlure thermique.

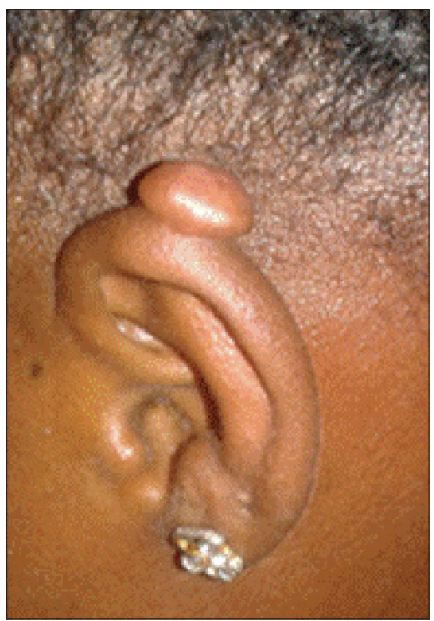

Figura 5: Chéloïde du pavillon de l'oreille gauche post piercing.

En effet, les progrès de ces dernières années ont permis d'identifier de nombreux facteurs physiopathologiques dont le Transforming Growth Factor (TGF $\beta$ ) et les gènes sur les chromosomes 2 et 7 à transmission autosomique dominante, avec pénétrance clinique incomplète et expression variable [24]. Ces modifications génétiques qui semblent s'exprimer au niveau de la régulation de la synthèse du collagène pourraient justifier cette prédisposition familiale. La longue durée d'évolution était probablement liée au caractère asymptomatique et bénin des lésions.

Sur le plan sémiologique, nos données étaient également proches de celles de la littérature en dehors de la taille des lésions qui étaient relativement plus petite et de l'aspect en pattes de crabes qui était peu retrouvé. Les tailles relativement petites, étaient en rapport avec les types de traumatismes retrouvés dans cette série. Il s'agissait en effet de petites lésions survenues lors des jeux, des bagarres et des AVP. La 
localisation préférentielle aux membres supérieurs et inférieurs était aussi en rapport avec le type de traumatisme, les membres étant les plus touchés lors des bagarres, des jeux et des chutes [6].

\section{Données Thérapeutiques}

Le peu de recours à la prise en charge médicale était également rapporté par Kibadi et al. [25] dont les travaux montraient que $79 \%$ des patients n'avaient prévu aucune démarche thérapeutique. Les raisons étaient entre autres le manque de moyens financiers, l'aggravation des lésions après toute tentative thérapeutique et la croyance selon laquelle la chéloïde serait héréditaire, donc incurable. Labsence de signes fonctionnels, la taille relativement petite des lésions, leurs localisations et leur nombre unique, pourraient expliquer l'absence de demande de soins médicaux. Il faut noter que dans nos régions, le traitement traditionnel est pratiqué en premier lieu, en deuxième lieu vient le recours à un paramédical qui est plus accessible et enfin en $3^{\text {ème }}$ position la consultation d'un médecin (référence). Pourtant les résultats montraient que le traitement médical avait entrainé un affaissement partiel ou total des lésions, contrairement au traitement traditionnel qui n'avait donné aucun résultat. Néanmoins la réalité dans la prise en charge des chéloïdes se confronte à la multiplicité des traitements qui témoigne de leur inefficacité. Antoine Petit dans son article intitulé «Chéloïdes: de l'offre au vocabulaire» décrit le refus de prise en charge des chéloïdes par les jeunes dermatologues français par manque d'expérience entre autres, il attribue également ces difficultés au qualificatif de cicatrice attribué aux chéloïdes [26].

\section{Données Sur Les Connaissances Et Le Retentissement}

Les ch/CH retentissaient peu sur la qualité de vie des lycéens et un retentissement esthétique était retrouvé surtout chez les filles, tout cela expliquant également le peu de recours à une consultation médicale. La méconnaissance des ch/CH par la moitié des élèves et la croyance en une origine mystique nous interpelle sur la nécessité de mener des campagnes d'information et sensibilisation sur les affections dermatologiques.

\section{CONCLUSION}

Les ch/CH sont fréquentes en milieu scolaire secondaire dans la ville de Ouagadougou. Elles atteignaient indifféremment les garçons et les filles. Létiologie traumatique était la plus retrouvée avec surtout les AVP. Les membres supérieurs et inférieurs étaient les localisations préférentielles. La fréquence élevée des AVP comme cause des cicatrices chéloïde/hypertrophiques, pourrait s'expliquer par le fait que les élèves de la ville de Ouagadougou ont comme principal moyen déplacement les engins à deux roues. Labsence de recours aux soins médicaux est probablement liée à l'ignorance des élèves sur les possibilités de prise en charge.

\section{Statement of Human and Animal Rights}

All procedures followed were in accordance with the ethical standards of the responsible committee on human experimentation (institutional and national) and with the Helsinki Declaration of 1975, as revised in 2008 .

\section{Statement of Informed Consent}

Informed consent was obtained from all patients for being included in the study.

\section{REFERENCES}

1. Pitche P. Quelle est la réalité des chélö̈des «spontanées» ?. Ann Dermatol Venereol. 2006;133:501.

2. Mostinckx S, Vanhooteghem O, Richert B, De La Brassinne M. Chéloïde et cicatrice hypertrophique. Ann Dermatol Venereal. 2005;132:384-7.

3. Beogo R, Guiébré YMC, Sérémé M, Ouoba K, Zwetyenga N. Cicatrices chélö̈des de la tête et du cou. Rev Stomatol Chit Maxillofac. 2012;113:179-83.

4. Datubo-Brown DD. Keloids: A review of the literature. Br J Plast Surg. 1990;43:70-7.

5. Di Cesare PE, Cheung DT, Perelman N, Libaw E, Peng L, Nimni ME. Alteration of collagen composition and cross-linking in keloid tissues. Matrix. 1990;10:172-8.

6. Gahongayire F, Simonart T, Heenen M, Paku M. Etiologies des chéloïdes à Kinshasa (République Démocratique du Congo): étude rétrospective de 768 malades). Ann Dermatol Venereol 2002;129:1174-8.

7. Murray JC, Pollack SV, Pinnell SR. Keloids: a review. J Am Acad Dermatol. 1981;4:461-70.

8. O'Sullivan ST, O'Shaughnessy M, O'Connor TPF. Aetiology and management of hypertrophic scars and keloids. Ann R Coll Surg Engl. 1996;78:168-75.

9. Ribault L, Martin JP, Larroque G. Les cicatrices chéloïdes cervicofaciales: à propos de 81 cas traités à Dakar. Ann Chir Plast Esthet. 1992;37:202-6.

10. Ud-Din S, Bayat A. Strategic management of keloid disease in ethnic skin: a structured approach supported by emerging litterature. $\mathrm{Br}$ Dermatol. 2013;169:71-81.

11. Retz. Des maladies de la peau et de celles de l'esprit (telles que les vapeurs, la mélancolie, la manie etc.), qui procèdent des affections du foie. Leur origine, la description de celles qui sont le moins connues, les traitements q ui leur conviennent. $3^{\text {eme }}$ ed. Paris: chez Méquignon, l’aîné; 1790: 155-8. 


\section{www.odermatol.com}

12. Alibert JM. Description des maladies de la peau observées à l'hôpital Saint Louis et exposition des meilleures méthodes suivies pour le traitement. 4eme édition. Paris: Barrois l'ainé et fils, 1806:113.

13. Bayat A, McGrouther DA, Ferguson MWJ. Skin scarring. BMJ. 2003;326:88-92.

14. Assi K, Kouame K, Ecra E, Gbery IP, Yoboue YP, Kanga JM. Aspects thérapeutiques des cicatrices chéloidiennes au centre de dermatologie du centre hospitalier et universitaire de Treichville (Abidjan CI). Mali Med. 2009;24:39-41.

15. Boui M, Lemnaouer A. Expérience dermatologique de l'hôpital marocain de campagne à Brazzaville, Congo. Med Trop. 2008;69:13-7.

16. Pitché P, Kombaté K, Barruet K, Tchangaï-Walla K. Cicatrices chéloïdes géantes. Ann Dermatol Venereol. 2000;127:333.

17. Niang SO, Sankale AA, Fall F, Diallo M, Dieng MT, Kane A. La place de la chirurgie dans la prise en charge des chéloïdes à Dakar. Med d'Afr Noire. 2009;5604:218-20.

18. Adegbidi H, Atadokpede F, do Ango-Padonou F, Yedomon H. Keloid acne of the neck: epidemiological studies over 10 years. Int J Dermatol. 2005;44:49-50.

19. Ouedraogo S. Analyse descriptive des facteurs associés à la survenue de chéloïdes sur peau noire au CHU YO [Thèse: Med]. Ouagadougou: Faculté de médecine, Université Saint Thomas d'Aquin;2014:100.

20. Institut Nationale de la Statistique et de la Démographie (INSD). Recensement général de la population et de l'habitat (RGPH). Burkina Faso (Projections 2017): Rapport commandité par le
Ministère de l'Administration Territoriale, de la Décentralisation et de la Cohésion sociale.

21. Annuaire statistique de l'enseignement secondaire du Burkina Faso 2015-2016. Commandité par la Direction des statistiques du Ministère de l'Education Nationale et l'Alphabétisation du Burkina Faso.

22. Cosman B, Crikelair F, Gaulin JC, Lattes R. The surgical treatment of keloids. Plast Reconstr Surg. 1961;24:335-58.

23. Traore SS, Bonkoungou G, Kirakoya B, Traore A, Zida M, Sanou A. Les chéloïdes: à propos de 52 cas traités à Ouagadougou. Ann Chir. 2000;125:803-5.

24. Laurent M. La cicatrice chéloïde: étude rétrospective sur 15 ans [Thèse: Med]. Nantes: Faculté de médecine, Université de Nantes;2007:93.

25. Kibadi K, Muhota DK, Mudimisi FN, Mufasoni SM, Mukendi YMW, Mukendi AT et al. Chéloïdes: aspects épidémiologiques et raisons du refus du traitement chirurgical à Kinshasa (République démocratique du Congo). Méd Trop. 2012;22:182-6.

26. Petit A. Chéloïdes: de l'offre de soins au vocabulaire Ann Dermatol Venereol. 2014:141:489-90.

Copyright by Adama Traoré, et al. This is an open-access article distributed under the terms of the Creative Commons Attribution License, which permits unrestricted use, distribution, and reproduction in any medium, provided the original author and source are credited.

Source of Support: Nil, Conflict of Interest: None declared. 